\title{
FRACTIONAL DIFFERENTIATION OF FUNCTIONS WITH LACUNARY FOURIER SERIES ${ }^{1}$
}

\author{
G. V. WELLAND ${ }^{2}$
}

1. This paper gives some results of a special nature for functions which have lacunary Fourier series. Before stating these, we begin with some definitions and comments.

Let $f:(0,2 \pi) \rightarrow R$ be integrable and assume it has the Fourier series

$$
S(f)=\frac{a_{0}}{2}+\sum_{k=1}^{\infty} a_{k} \cos n_{k} x+b_{k} \sin n_{k} x
$$

$f$ is said to have a lacunary Fourier series if there is a $\lambda>1$ such that for $k=1,2, \cdots$ we have $n_{k+1}>\lambda n_{k}$. We will assume $a_{0}=0$. Sometimes we will prefer to write $S(f)$ in its complex form

$$
S(f)=\sum_{-\infty}^{+\infty} C_{k} \exp \left(i n_{k} x\right)
$$

where $\sum^{\prime}$ indicates that the sum excludes the term $k=0$.

Let $0 \leqq k<\alpha<k+1$ and $\alpha+\beta=k+1$, with $k$ an integer, be given. We define the $\beta$ th integral of $f$ by

$$
F(x)=f_{\beta}(x)=\frac{1}{\Gamma(\beta)} \int_{-\infty}^{x} f(t)(x-t)^{\beta-1} d t .
$$

A discussion of this integral is given in [6, page 133]. There it is shown that

$$
S\left(f_{\beta}\right)=\sum_{-\infty}^{\infty} \frac{C_{k}}{\left(i n_{k}\right)^{\beta}} \exp \left(i n_{k} x\right),
$$

and it is pointed out that the convergence of the integral is bound up with the fact that $a_{0}=0$. From this it is clear that $F$ has a lacunary Fourier series if $f$ does. $f$ is said to have an $\alpha$ th derivative if $F$ has a $k+1$ Peano derivative at $x_{0}$, i.e., there exists a polynomial $P_{x_{0}}(t)$ of degree $\leqq k+1$ such that

$$
F\left(x_{0}+t\right)-P_{x_{0}}(t)=o\left(|t|^{k+1}\right), \quad t \rightarrow 0 .
$$

If

Received by the editors November 30, 1966.

1 This is a portion of the author's doctoral dissertation at Purdue University.

2 Partially supported by NSF Grant GP-1665. 


$$
\begin{aligned}
\left\{\frac{1}{\rho} \int_{-\rho}^{\rho}\left|F\left(x_{0}+t\right)-P_{x_{0}}(t)\right| p d t\right\}^{1 / p} & =o\left(\rho^{k+1}\right), \\
& \rho \rightarrow 0 \quad(1 \leqq p<\infty),
\end{aligned}
$$

$f$ is said to have an $\alpha$ th derivative in the $L^{p}$ sense. If there exists a polynomial $Q_{x_{0}}(t)$ of degree $\leqq k$ such that $R_{x_{0}}(t)=f\left(x_{0}+t\right)-Q_{x_{0}}(t)$ $=O\left(|t|^{\alpha}\right)$ as $t \rightarrow 0$ then $f$ is said to satisfy the condition $\Lambda_{\alpha}$ and if

$$
\left\{\frac{1}{\rho} \int_{-\rho}^{\rho}\left|R_{x_{0}}(t)\right|^{p} d t\right\}^{1 / p}=O\left(\rho^{\alpha}\right), \quad \rho \rightarrow 0 \quad(1 \leqq p<\infty),
$$

$f$ is said to satisfy the condition $\Lambda_{\alpha}^{p}$. $f$ is said to satisfy the condition $N_{\alpha}^{p}$ if for some $\rho>0$

$$
\frac{1}{\rho} \int_{-\rho}^{\rho} \frac{\left|R_{x_{0}}(t)\right|^{p}}{|t|^{1+p \alpha}} d t<\infty .
$$

If $\alpha=1$, the condition $\Lambda_{1}$ is replaced by the condition $\Lambda_{*}$, which requires that $\Delta^{2} f\left(x_{0}, t\right)=f\left(x_{0}+t\right)+f\left(x_{0}-t\right)-2 f\left(x_{0}\right)=O(t)$ as $t \rightarrow 0$. Finally, $f$ is said to have an approximate derivative at $x_{0}$, equal to $f_{a p}^{\prime}\left(x_{0}\right)$, if $\left|f\left(x_{0}+t\right)-f\left(x_{0}\right)-f_{a p}^{\prime}\left(x_{0}\right) t\right|=o(t)$ as $t \rightarrow 0$ through a set having zero as a point of density.

Throughout, constants depending only on the parameters of the problem will be denoted by $D_{k}, D, M, C$ etc. These will not necessarily denote the same constant at each point.

The results we will state were motivated by the main result in [4] which gives the following theorem for the case $p=2$.

THEOREM A. The necessary and sufficient condition for $f$ to satisfy the conditions $N_{\alpha}^{p}, N_{\alpha}^{2}(2 \leqq p<\infty)$ almost everywhere in a measurable set $E$ is that $f$ satisfy the condition $\Lambda_{\alpha}^{p}$ and have an $\alpha$ th derivative in the $L^{p}$ sense almost everywhere in the set $E$.

The proof of this theorem follows the proof of the theorem in [4] with $p=2$ and a few minor modifications. The results in [4] parallel the results of [3] on differentiability of functions.

We are now ready to state the two theorems of this paper.

THEOREM 1. Let $f$ have a lacunary Fourier series and suppose $f$ has an $\alpha$ th derivative at some point $x_{0}(0 \leqq k<\alpha<k+1)$. Then $f$ satisfies the condition $\Lambda_{\alpha}$ everywhere.

THEOREM 2. Let $F$ be a function with a lacunary Fourier series. If $\sum\left(a_{j}^{2}+b_{j}^{2}\right) n_{j}^{2}<\infty$ then $F$ has a derivative almost everywhere.

The first of these is proved by a method similar to one in [5] which 
is adapted to higher orders of differentiation by means of Taylor's theorem. The second is a corollary of the main theorem in [3]. It is included here because of some if its corollaries.

2. We start with the proof of Theorem 1 . We may assume $x_{0}=0$ and, under the assumption that the polynomial in (3) is identically zero, we have $F\left(x_{0}+t\right)=F(t)=R(t)$ where $R(t)=O\left(|t|^{k+1}\right)$ as $t \rightarrow 0$. Let $N=N_{j}=\min \left\{n_{j}-n_{j-1}, n_{j+1}-n_{j}\right\}$. We can choose an integer $p>0$ such that $N-1<p(k+2) \leqq N+k+1$. Let

$$
K^{j}(t)=K(t)=B_{k}^{-1}\left(\frac{\sin (p t / 2)}{p \sin (t / 2)}\right)^{2(k+2)},
$$

where

$$
B_{k}=\frac{1}{\pi} \int_{-\pi}^{\pi}\left(\frac{\sin (p t / 2)}{p \sin (t / 2)}\right)^{2(k+2)} d t .
$$

$K(t)$ is a trigonometric polynomial of degree $(p-1)(k+2)$ and $(1 / \pi) \int_{-\pi}^{\pi} K(t) d t=1$. Hence we may write $K(t)=1 / 2+\sum_{\sigma=1}^{N-1} d_{\epsilon} \cos \sigma t$ where

$$
d_{\sigma}=\frac{1}{\pi} \int_{-\pi}^{\pi} K(t) \cos \sigma t d t \quad \sigma=1,2, \cdots, N-1 .
$$

Because

$$
\begin{aligned}
B_{k} & =\frac{1}{\pi} \int_{-\pi}^{\pi}\left(\frac{\sin (p t / 2)}{p \sin (t / 2)}\right)^{2(k+2)} d t>\frac{2}{\pi} \int_{0}^{1 / p}\left(\frac{\sin (p t / 2)}{p \sin (t / 2)}\right)^{2 k+4} d t \\
& \geqq \frac{1}{p}\left(\frac{2}{\pi}\right)^{2 k+5}=\frac{1}{p} D_{k},
\end{aligned}
$$

it can be shown that

$$
\begin{aligned}
K(t) & \leqq D_{k}^{\prime} p, \quad 0 \leqq|t| \leqq 1 / N, \\
& \leqq D_{k}^{\prime}\left(1 / p^{k+2}|t|^{k+3}\right), \quad 1 / N \leqq|t| \leqq \pi,
\end{aligned}
$$

where we recall $k<\alpha<k+1$ is fixed.

It is easy to show that

$$
\begin{aligned}
& 2 K(t) \cos n_{j} t=\cos n_{j} t+\sum_{\sigma=1}^{N-1} d_{\sigma}\left[\cos \left(n_{j}+\sigma\right) t+\cos \left(n_{j}-\sigma\right) t\right], \\
& 2 K(t) \sin n_{j} t=\sin n_{j} t+\sum_{\sigma=1}^{N-1} d_{\sigma}\left[\sin \left(n_{j}+\sigma\right) t+\sin \left(n_{j}-\sigma\right) t\right] .
\end{aligned}
$$

Since $n_{j-1}<n_{j}-\sigma<n_{j}+\sigma<n_{j+1}$ for $\sigma=1,2, \cdots, N-1$, we have 


$$
\left(a_{j}, b_{j}\right)=\frac{1}{\pi} \int_{-\pi}^{\pi} F(t) 2 K(t)\left(\cos n_{j} t, \sin n_{j} t\right) d t,
$$

where $a_{j}, b_{j}$ are the Fourier coefficients of $F$.

Let $\epsilon>0$ be given. Then there exists a $\delta>0$ such that for $|x|<\delta$ $|F(x)|<\epsilon|x|^{k+1}$ since $f$ has an $\alpha$ th derivative at $x=0$. Assuming $1 / N<\delta$ and applying formula (4) we obtain

$$
\begin{aligned}
\left|a_{j}\right| \leqq & \frac{2}{\pi} \int_{0}^{\pi}|F(x)| K(x) d x \leqq D_{k}^{\prime} N^{\prime} \epsilon \int_{0<|x| \leqq 1 / N}|x|^{k+1} d x \\
& +D_{k}^{\prime} N^{-(k+2)} \epsilon \int_{1 / N \leqq|x| \leqq \delta}|x|^{-2} d x \\
& +D_{k}^{\prime} N^{-(k+2)} \int_{\delta \leqq|x| \leqq \pi}|F(x)| \cdot|x|^{-(k+3)} d x \\
\leqq & M_{1} \epsilon N^{-(k+1)}+M_{2} \delta^{-(k+3)} N^{-(k+2)} \int_{-\pi}^{\pi}|F(x)| d x \\
\leqq & M_{1}(L)^{k+1} \epsilon\left(n_{j}\right)^{-(k+1)}+M_{3} \frac{\delta^{-(k+3)}}{n_{j}}(L)^{k+2}\left(\frac{1}{n j}\right)^{k+1}
\end{aligned}
$$

where $M_{1}, M_{2}$ and $M_{3}$ are constants and $L=\min \{\lambda-1,1-1 / \lambda\}$. Since $M_{1}, M_{2}, M_{3}$ and $L$ are all fixed constants and we can choose $j$ so large that $M_{3} \delta^{-(k+3)}<n_{j} \epsilon$, we see that $a_{j}=o\left(n_{j}^{-(k+1)}\right)$ as $j \rightarrow \infty$. Similarly, $b_{j}=o\left(n_{j}^{-(k+1)}\right)$ as $j \rightarrow \infty$.

If we denote by $c_{j}(F)$ and $c_{j}(f)$ the Fourier coefficients of $F$ and $f$ respectively we see that $c_{j}(F)=o\left(n_{j}^{-(k+1)}\right)$ as $|j| \rightarrow \infty$ and hence that $c_{j}(f)=o\left(n_{j}^{-(k+1)+\beta}\right)=o\left(n_{j}^{-\alpha}\right)$ as $|j| \rightarrow \infty$. A result in [2] shows that for $0<\alpha<1$ we have $f$ satisfies the condition $\Lambda_{\alpha}$ if and only if $c_{j}$ $=O\left(n_{j}^{-\alpha}\right)$.

Suppose that $k<\alpha<k+1$. Then the series

$$
\sum_{-\infty}^{\infty} c_{j}\left(i n_{j}\right)^{k} \exp (i n, t)
$$

converges uniformly. To see this, let $\epsilon>0$ be given. Then there exists $M>0$ such that $\left|c_{j}\right| \leqq M n_{j}^{-\alpha}, \quad j=\mp 1, \mp 2, \cdots$. Choose $N$ such that for $|j| \geqq N,\left|n_{j}\right|^{\beta-1} \leqq C \epsilon$, where $C^{-1}=2 M \sum_{1}^{\infty}\left(\lambda^{\beta-1}\right)^{j}$. Then we see that

$$
\begin{aligned}
\sum_{|j| \geq N}\left|c_{j}\right| \cdot\left|n_{j}\right|^{k} & \leqq \sum_{|j| \geqq N} M \cdot\left|n_{j}\right|^{-\alpha} \cdot\left|n_{j}\right|^{k} \\
& \leqq 2 M\left|n_{N}\right|^{\beta-1} \sum_{=N}\left(\lambda^{\beta-1}\right)^{j} \leqq C^{-1} C \epsilon=\epsilon .
\end{aligned}
$$


This shows that $f$ has $k$ derivatives and that the $k$ th derivative has coefficients which are $o\left(n_{j}{ }^{\beta-1}\right)$ as $j \rightarrow \infty$, and hence $f^{k}(x)$ satisfies the condition $\Lambda_{1-\beta}$.

We now apply Taylor's theorem with remainder to see that

$$
f(x+t)=\sum_{j=1}^{k} f^{j}(x) \frac{t^{3}}{j !}+R(t)
$$

where

$$
R(t)=\frac{1}{(k-1) !} \int_{0}^{t}(t-u)^{k-1}\left[f^{k}(x+u)-f^{k}(u)\right] d u .
$$

Since $f^{k}(x)$ satisfies $\Lambda_{1-\beta}$, it follows that $f(x)$ is in $\Lambda_{\alpha}$ because

$$
R(t)=O\left(\int_{0}^{t}(t-u)^{k-1} u^{1-\beta} d u\right)=O\left(|t|^{k+1-\beta}\right)=O\left(|t|^{\alpha}\right), \quad t \rightarrow 0 .
$$

Finally, suppose that the polynomial in (3) is not identically zero, then we can find a $C_{0}^{\infty}$ function $g$ which has this polynomial as its Taylor's expansion up to the $(k+1)$ th term at the point $x=0$ and with support in $(-\pi, \pi)$. It is easy to see that $g$ has Fourier coefficients which are $O\left(n^{-(k+3)}\right)$. Let $G=F-g$ and let $F$ be replaced by $G$ in the integral formula (4). Then $\left|a_{j}\right|$ is dominated by that integral $+O\left(\sum_{n_{j}}^{n_{j+1}}\left|a_{j}(g)\right|\right)$ where $a_{j}(g)$ are the Fourier coefficients of $g$. The integral is $O\left(n_{j}^{-(k+1)}\right)$ as before and

$$
\begin{aligned}
\sum_{n_{j}}^{n_{j}+1}\left|a_{j}(g)\right| & =O\left(\sum_{n_{j}}^{\infty} n_{j}^{-(k+3)}\right)=O\left(n_{j}^{-(k+1)} \sum_{n_{j}} n^{-2}\right) \\
& =o\left(n_{j}^{-(k+1)}\right), \quad j \rightarrow \infty .
\end{aligned}
$$

Hence $\left|a_{j}\right|=o\left(n_{j}^{-(k+1)}\right)$. This completes the proof of Theorem 1 .

3. To begin the proof of Theorem 2 we note that because of the main result in [3] we only need to show that $f$ satisfies the condition $\Lambda_{*}$ and that the integral

$$
\int_{-\pi}^{\pi} \frac{\left|\Delta^{2} f(x, t)\right|^{2}}{|t|^{3}} d t<\infty \quad \text { for almost all } x .
$$

However, since $a_{j}, b_{j}=O\left(n_{j}\right)$ as $j \rightarrow \infty$, it can be shown using the technique of $\left[6\right.$, Volume I, page 47] that $f$ satisfies the condition $\Lambda_{*}$.

We show the integral (5) is finite for almost every $x$ in $[-\pi, \pi]$ by showing the following integral is finite 


$$
\int_{-\pi}^{\pi} \int_{-\pi}^{\pi} \frac{\left|\Delta^{2} f(x, t)\right|^{2}}{|t|^{3}} d t d x
$$

This is equal to

$$
\int_{-\pi}^{\pi} \int_{-\pi}^{\pi} \frac{1}{|t|^{3}}\left(-\sum\left[a_{k} \cos n_{k} x+b_{k} \sin n_{k} x\right] 4 \sin ^{2}\left(\frac{n_{k} t}{2}\right)\right)^{2} d t d x
$$

By Parseval's equality this is

$$
16 \int_{-\pi}^{\pi}\left(\sum\left(a_{k}^{2}+b_{k}^{2}\right) \sin ^{4}\left(\frac{n_{k} t}{2}\right)\right) \frac{d t}{|t|^{3}} .
$$

We have

$$
\begin{aligned}
\int_{-\pi}^{\pi} \sin ^{4}\left(\frac{n_{k} t}{2}\right) \frac{d t}{|t|^{3}} & =2 \int_{0}^{\pi / n_{k}} \sin ^{4}\left(\frac{n_{k} t}{2}\right) \frac{d t}{t^{3}}+2 \int_{\pi / n k}^{\pi} \sin ^{4}\left(\frac{n_{k} t}{2}\right) \frac{d t}{t^{3}} \\
& \leqq 2 \int_{0}^{\pi / n k}\left(\frac{n_{k} t}{2}\right)^{4} \frac{d t}{t^{3}}+2 \int_{\pi / n k}^{\pi} \frac{d t}{t^{3}} \\
& =\left(\frac{n_{k}}{2}\right)^{4} \cdot\left(\frac{\pi}{n_{k}}\right)^{2}+\left(\frac{n_{k}^{2}}{2}-\frac{1}{\pi^{2}}\right)=O\left(n_{k}^{2}\right),
\end{aligned}
$$

and hence the integral (6) is bounded by $16 D \sum_{k}\left(a_{k}^{2}+b_{\mathbf{k}}^{2}\right) n_{\mathbf{k}}^{2}<\infty$. This completes the proof of the theorem.

We add some corollaries.

Corollary 1. Suppose $f$ has a lacunary Fourier series. Then if $f$ has an approximate derivative in a set of positive measure $f$ has a derivative almost everywhere.

Proof. This follows from the fact (see e.g. [1, Volume II, page 263]) that if a function $f$ has a lacunary Fourier series which converges on a set of positive measure to a function which can be extended to an absolutely continuous one, then $\sum_{j}\left(a_{j}^{2}+b_{j}^{2}\right) n_{j}^{2}<\infty$.

Corollary 2. Let $0<\alpha<1, \alpha+\beta=1$, and $f$ have a lacunary Fourier series. Then $f$ has an $\alpha$ th derivative in a set $E$ of positive measure and hence almost everywhere if and only if for almost every $x$ in $E$ there is an $\eta_{x}=\eta>0$ such that

$$
\int_{-\eta}^{\eta} \frac{R_{x}(t)^{2}}{|t|^{1+2 \alpha}} d t<\infty
$$

Proof. Let $F(x)=f_{\beta}(x)$. Assuming (7), we have $F(x)$ has a derivative in the $L^{2}$ sense and hence an approximate derivative almost 
everywhere in $E$. By Corollary $1, F(x)$ has an ordinary derivative almost everywhere. On the other hand, if $f$ has an $\alpha$ th derivative almost everywhere in $E$, then $F(x)$ has an ordinary derivative almost everywhere again by Corollary 1 . By Theorem $1, f$ satisfies the condition $\Lambda_{\alpha}$ almost everywhere. Hence, by Theorem A, (7) holds almost everywhere.

This corollary extends to the case $\alpha>1$ by applying Taylor's theorem as in Theorem 1.

\section{BIBLIOGRAPHY}

1. N. K. Bari, A treatise on trigonometric series, Vols. I, II, translated from Russian, Macmillan, New York, 1964.

2. G. G. Lorentz, Fourier-Koeffizienten und Funktionenklassen, Math Z. 51 (1948), 135-149.

3. E. M. Stein and A. Zygmund, On the differentiability of functions, Studia Math. 23 (1964), 247-283.

4. - On the fractional differentiability of functions, Proc. London Math. Soc. (3) 14a (1965), 249-264.

5. Xie Ting-Fan, On lacunary Fourier series, Chinese Math. Acta 5 (1964), $340-345$.

6. A. Zygmund, Trigonometric series, Vols. I, II, 2nd ed., Cambridge Univ. Press, New York, 1959.

De Paul University 